\title{
A Practical Guide to the Measurement of Turbidity Curves of Cooling Crystallisations from Solution
}

Received 00th January 20xx, Accepted 00th January 20xx

DOI: 10.1039/x0xx00000 $\mathrm{x}$

\author{
Simon J. Coles ${ }^{\mathrm{a}}$ and Terence L. Threlfall*a
}

Several methods for the determination of the temperatures of crystallisation, crystal proliferation and crystal dissolution in cooling crystallisations are presented and compared. These include curve fitting, statistical and visual determination. It is shown that the quick analyses are as reproducible as the longer, mathematically based methods. The point of crystal proliferation is suggested to be the more useful, more meaningful, more reproducible and more quickly determined piece of information than the universally recommended point of first crystal detection.

\section{Introduction}

We have been unable to find, either in the scientific literature or in instrument manufacturers' publications, any account of the practicalities of the determination of the crystallisation point of cooling solutions by turbidimetry, despite the widespread use of turbidimetry to monitor crystallisation ${ }^{1-2}$. It must therefore be supposed that it is regarded as a trivial matter, unworthy of comment. Indeed, if the baseline could be aligned to be perfectly horizontal and free from noise, the determination of the first deviation from that level base would be easy and unambiguous. Ordinary solutions, before careful filtration, typically contain $10^{6}$ particles per cubic metre ${ }^{3}$, and even after ultrafiltration no solution can be totally free of particles. The contribution from the stochastic nature of crystallisation ${ }^{4}$ as well as the random noise of the baseline, due both to extraneous particles and to the electronic noise in the measurement system, must be taken into consideration in assessing the reproducibility of the crystallisation point. There is also the question of what is actually required to be measured. Nucleation is hardly determinable, certainly not turbidimetrically, so

\footnotetext{
a. School of Chemistry, Faculty of Engineering and Physical Sciences, University of Southampton, Southampton, UK. SO17 1BJ.

*Email t.threlfall@soton.ac.uk
}

Electronic Supplementary Information (ESI) available: [details of any supplementary information available should be included here]. See DOI: 10.1039/x0xx00000x the first detectable crystallisation point is commonly measured. This is then dependent on the sensitivity of the detection system. However, it will be argued here that what is really required is the point of crystal proliferation. Methods of determining this will also be described.

It is generally considered that the temperature of dissolution should be capable of more precise measurement than that of crystallisation. Our experience has shown that the variation in the dissolution point is always much larger than would be expected from consideration of the solubility as a thermodynamic quantity ${ }^{5-7}$. This variation, as measured in temperature terms, can always be reduced by slowing the rate of heating for a solution uncomplicated by polymorphism. The question then arises as to how representative those conditions will be in relation to solution processing, for which these measurements are a necessary preliminary.

The turbidity measured in cooling crystallising of solutions is generally due to particle obscuration rather than to diffraction phenomena ${ }^{8}$. This is a result of the particles being of several micrometers and upwards in diameter which are involved ${ }^{9-11 \ddagger}$. Analyses of turbidity during reactive crystallisation have been noted ${ }^{12}$, but this results in smaller particles and plays no part in the investigations upon which this paper is based.

We were concerned by the variation in crystallisation and dissolution temperatures that were recorded during a range of 
crystallisation studies performed in our laboratory, particularly for aqueous D, L-malic acid solutions, and decided to compare various methods of assessment. The present paper is the result of applying these comparisons to over a thousand crystallisations of many different crystallising solutions, mainly in aqueous or aqueous ethanolic media. The comparisons should therefore apply generally, rather than being confined to the systems described. The experimental which follows merely describes the origin of the charts used to illustrate the various procedures. Although this paper is presented as an elementary practical guide, it is hoped that it will be found useful to any experimenter, even an experienced one.

\section{Experimental}

The crystallisation of solutions was monitored turbidimetrically and where appropriate checked visually under cross-illumination. The temperature ramps were provided by a programmable water-bath to a jacketted crystallisation vessel. A typical set-up is shown in Figure 1.

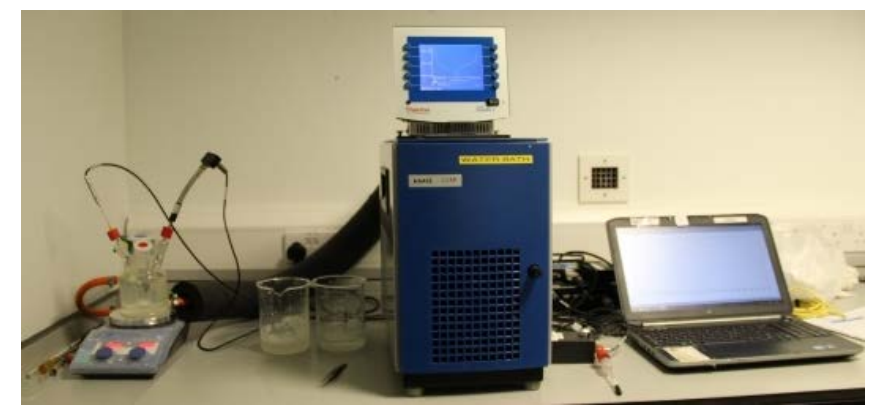

Figure 1. The water bath in the middle provides the temperature control of the magnetically stirred crystallising vessel on the left via circulating water to the jacket. The turbidity and temperature probes in the vessel send data to the computer on the right.

The dissolution point was determined as the temperature of the final disappearance of turbidity. The crystallisation was determined as the temperature of first detection of turbidity, or as that of the sudden increase of turbidity (see discussion). The measurements presented here were made on an aqueous magnesium sulfate solution, which is believed to produce ${ }^{\ddagger \neq}$ solely the heptahydrate provided the temperature is kept below $46^{\circ} \mathrm{C}^{11}$, or on aqueous DL-malic acid solutions which display complicated crystallisation behaviour. Transformation from a metastable form to the stable form can and does occur during crystallisation, so the dissolution point depends on the preceding ramp ranges and rates as well as on the usual factors considered relevant to the determination of dissolution points. Because the solid-solid state nucleation of the stable polymorph is as erratic as that of nucleation from the solution, the dissolution point is also liable to be variable. Coincidentally, L-malic acid shows similar behaviour if the temperature ramps are suitable, again showing two turbidity peaks.

The crystallisation of DL-malic acid shows further complexity, namely the presence of a third polymorph with reversed solubility, (more soluble cold than hot), which it is intended to describe in detail in a subsequent paper. Only those issues relevant to the determination of crystallisation and dissolution will be presented here.

All the measurements presented herein were obtained using an HEL E1041S turbidity probe operated by CrystalEyes software, and with either a ThermoHaake C25P or a Julabo F25 programmable water bath. Further turbidity measurements were made using two turbidity probes, one of standard transflectance design and one of a roof-prism design in a Cambridge Reactor Design 'Polar Bear Plus' crystalliser system. These gave similar results to those presented in the charts here. None of those results are shown here. The jacketted crystallising vessels have been described previously ${ }^{12}$. The cooling and heating ramps were set at $1^{\circ}$ per hour or $3^{\circ}$ per hour around the crystallisation and dissolution temperatures respectively. However they were performed more rapidly in between these points, typically at $10^{\circ}$ per hour so as to reduce the overall cycle time. The data were transferred to Microsoft Excel, converted to graphs and analysed. By 'crystallisation temperature' is implied the first detectable increase in turbidity deemed to be due to the presence of crystals in the solution. By 'onset of secondary nucleation' is implied the point at which the turbidity rises significantly and increasingly giving rise to an elbow in the turbidity curve. The term 'onset of cryst proliferation' is identical, but is more frequent in this paper because we are concerned with phenomena rather than mechanisms, except where these are observable rather than implied. 'Heterogeneous nucleation' implies nucleation ascribable to the presence of foreign bodies in the solution ${ }^{13}$. 
It will be realized that the succeeding discussion is a very general one, applicable to any solution crystallising under any conditions and also, mutus mutandi, to any detection system. The purpose of this experimental section is merely to provide some experimentally generated turbidity graphs to illustrate the methods of measurement and the possible problems which might be encountered.

\section{Results and discussion}

In each of the charts, the temperature ramp is in blue and the turbidity is in orange whenever only a single crystallisation run is represented. The turbidity is the arbitrary scale of the turbidity detection system.

The curve in Figure 2 results from the unseeded cooling crystallisation of aqueous D, L-malic acid solution at $1^{\circ}$ per hour, with turbidity recorded every 20 seconds.

\section{INSERT FIGURE 2 HERE}

Figure 2. Typical turbidity curve in orange, heating and cooling cycle in blue. Turbidity is in arbitrary units.

The baseline values begin to rise due to the presence of detectable seeds from heterogeneous nucleation. These values continue to increase due to the growth of those seeds, because turbidity is proportional to the surface area ${ }^{6,14 \S}$. This increase in turbidity is entirely due to crystal growth on heterogeneously generated seeds. That this portion of the curve is due to crystal growth unaccompanied by new nucleation can easily be verified by seeding such solutions with a small number of large crystals. Observing the subsequent behaviour of the seeds in the solution visually confirms that the growing crystals must be the cause of the increase of recorded turbidity. This behaviour is consequent on the solution being in the growthonly zone of the metastable zone ${ }^{4}$. Existing crystals grow, but new ones do not form. That unseeded crystallisations show the same behaviour as seeded nucleation is due to the initial crystallisation being dependent on heterogenous nuclei: once all the active particles have initiated nucleation events there is no further source of fresh nucleation. During further cooling the Secondary Nucleation Threshold is reached. At this point the crystals start to proliferate, a matter which also can be verified by visual observation. The turbidity curve begins to rise more quickly at this point.

\section{Determining the temperature at which crystal proliferation begins}

How does one determine this point of secondary nucleation?

Because turbidity is proportional to surface area $^{14}$, the onset of proliferation should force the turbidity to begin to follow a cubic curve instead of the previous quadratic one, since the turbidity is now responding to the mass of crystals. In Figure 3, the initial experimental turbidity curve is fitted by a quadratic function and the next portion by a cubic function.

\section{INSERT FIGURE 3 HERE}

Figure 3. Curve fitting of the experimental turbidity rise (blue line) associated with nucleation and crystallisation. Point of intersection $=358.5,0.01$; Temperature $=$ $19.444^{\circ} \mathrm{C}$. The equations were generated from a curvefitting program in Microsoft Excel, see text for details.

The calculated green curve is a quadratic representing the period of crystal growth. The calculated orange curve is based on a cubic equation representing the crystal proliferation region. The summation of these two curves is shown as the purple curve, which closely follows the experimental curve. All the lines and equations of figure 3 were generated using the polynomial option in the Trendline routine in Microsoft Excel, accessible via the chart elements tab generated by clicking on the chart. The second order curve representing the first part of the crystallisation was first calculated. This curve was then subtracted from the experimental curve to check that that part of the curve could accurately be represented by a third order function but not by a second order. The two computed curves were then added to give the purple curve. Some judgement is required as to the chosen range in order to generate meaningful results. At higher turbidities, aggregation, agglomeration ${ }^{15}$ and multiple scattering combine with detector limits to depress the turbidity curve. The crossing point of the quadratic and cubic equations at $19.44^{\circ} \mathrm{C}$ represents the onset of crystal proliferation at the Secondary Nucleation Threshold. 
The complexity of the dense slurry at at higher turbidities means that there can be no expectation of meaningful curve analysis and none has been attempted for the remaining portion of the curve. As can be seen, a very good analysis of the curve from the baseline to well past the proliferation point can be achieved by considering the initial turbidity as a quadratic function and the subsequent part as a cubic one. No satisfactory curve fitting over the whole crystallisation region could be achieved by a single power equation. It may be objected that mathematics is so powerful that a multiplicity of functions would fit, so the analysis is meaningless. However, it will be found impossible to model both the initial rise from the baseline and the elbow by a single second order function (or third, or fourth order). Either the elbow is fitted when the initial rise cannot be, or vice versa as used here. This is true both of an equation of the form $y=x^{2}+c$ which is of the expected curvature, or even of the form $x=y^{2}+c$ which does not bear a meaningful relation to the events. The discrepancy at the elbow can be perfectly eliminated by a third order function. Two objections may now be raised. The first relates to the quadratic part, the second to the cubic part. Since the temperature is steadily reducing, the driving force and therefore the crystallising rate should be increasing. However, this is compensated by the fact that as the crystal grows, more molecules need to be added to achieve a constant increase in area. In any case, the temperature drop between first crystal detection and crystal proliferation is commonly only a small proportion of the metastable zone width. Experience of a large number of crystallising systems, including L-malic acid, ascorbic acid, glycine, mannitol, propionanilide, Rochelle salt and xylose, as well as the examples shown here of D,L-malic acid and magnesium sulfate indicates that the initial crystallisation can be closely modelled by a quadratic equation. The second objection relates to the versatility of the curvature in a cubic equation some point of the curve, indeed of any cubic curve may have the necessary curvature. This is a valid argument and we are not claiming that another function or a different part of the curve would not fit, only that the procedure here is not incompatible with the model of crystallisation. In fact, a straight line will give an intercept which is not significantly different to the analysis presented here for any reasonably slow cooling ramp. The quadratic followed by the cubic curve has the overwhelming advantage of being linked theoretically to the events taking place.

Of course, such an analysis of a crystallising curve is very tedious. We have however measured many turbidity curves both by that analysis and by eye, assessing the position of the elbow, or regarding the proliferation point as the turning point of a hyperbola with axes set at an angle to the crystallisation axes. Not only is this easier and quicker than curve fitting, it is also easier and quicker than determining the crystallisation point. From an analysis of 39 seeded Design of Experiment runs in triplicate on magnesium sulfate solution it also transpires that for a ramp rate of $1^{\circ} \mathrm{C}$ per hour, visual assessment gives values within $0.1^{\circ} \mathrm{C}$ of curve fitting, typically marginally higher in temperature. The repeatability of the visual measurement of the proliferation point from the chart, (or reproducibility from different observers) is consistently better than that of the determination of first crystallisation point, as can be seen from Table 1.

\begin{tabular}{|l|l|l|l|}
\hline $\begin{array}{l}\text { Detectio } \\
\mathrm{n}\end{array}$ & $\begin{array}{l}\text { Proliferatio } \\
\mathrm{n}\end{array}$ & $\begin{array}{l}\text { Dissolutio } \\
\mathrm{n}\end{array}$ & Source \\
\hline 1.09 & 0.83 & 0.81 & $\begin{array}{l}\text { 200 unseeded D,L- } \\
\text { malic acid cycles }\end{array}$ \\
\hline 0.62 & 0.30 & 0.60 & $\begin{array}{l}30 \text { seeded D,L- } \\
\text { malic acid cycles }\end{array}$ \\
\hline 0.42 & 0.29 & 0.10 & $\begin{array}{l}150 \quad \text { seeded } \\
\mathrm{MgSO}_{4} \text { cycles }\end{array}$ \\
\hline
\end{tabular}

Table 1. Comparative standard deviations in ${ }^{\circ} \mathrm{C}$ of the crystallisation detection, proliferation and dissolution temperatures from crystallisations of magnesium sulfate and $d, l-$ malic acid.

The average discrepancy of replicate measurements by visual assessment of the charts was $0.10^{\circ} \mathrm{C}$, whilst that by curve fitting was $0.09^{\circ} \mathrm{C}$. These figures show that visual assessment of the charts is as good a method of determining the proliferation point as curve fitting methods.

The deviation of measurements of repeat determinations is due to both the inherent reproducibility of the crystallisation events as well as to the errors of measurement. So it is not claimed that the measurement itself is more accurate, only that the reproducibility of the determination of the proliferation point by visual examination is better than that of the initial crystallisation point by examination of the 
change of turbidity in the tabulated data from which crystallisation charts, such as that of Figure 2, are derived. It may well be the case that the proliferation point is itself less variable than the first crystal detection point.

Of course, one may wish to determine the crystallisation point, but it is probably more meaningful in industrial practice to know the proliferation temperature than the temperature at which the first crystals appear. This latter temperature is in any case an arbitrary one dependent on nucleation, crystal growth, detectability and rate of temperature drop between nucleation and crystal detectability.

\section{Determining the temperature of first crystal detection}

If the baseline is very constant it may simply be possible to use the first noted rise away from that baseline to determine the point of crystallisation. This was the method used in a previous paper ${ }^{12}$. The manufacturer's default for the output of the turbidity is 2 decimal places on a turbidity scale of 5 units, but we have found that 3 decimal places is more useful. When there is noise on the baseline we now use the criterion of the first value for which there is no subsequent lower value. This is illustrated in Figure 4 below, where the value at point 264 fulfils that criterion.

\section{INSERT FIGURE 4 HERE}

Figure 4. Part of a turbidity curve showing the dip before crystallisation due to the effect of refractive index change of the solution before the crystallisation event. Both the extent of the reduction of turbidity and the depth of the dip in this chart are larger than normally encountered.

It may be necessary to combine this procedure with a moving point smoothing of the turbidity points. We commonly use 20-point average smoothing (AVERAGE function in Excel). This procedure for the assessment of crystallisation point either on unsmoothed or smoothed data is usually unambiguous, but doubts occasionally arise, perhaps in $2-3 \%$ of the cycles, where there appears to be a rogue value. If this appears unresolvable by judgement, it can always be decided by using averaging or averaging over a larger range of values. Because the curve is rising exponentially, smoothing from the mid-point of the range will shift the values along - see Figure 5, in which the distortion of the curve can be seen and in which no smoothing yielded a value of $18.76^{\circ} \mathrm{C}$ for the initial crystallisation temperature (20-point smoothing, $18.81^{\circ} \mathrm{C}$; 50-point smoothing, 18.99; 80 -point smoothing, $19.09^{\circ} \mathrm{C}$ ).

\section{INSERT FIGURE 5 HERE}

Figure 5. Excessive smoothing: some smoothing may assist in the determination of the first detectable crystallisation. However, the danger of excessive smoothing on the apparent start of crystallisation is shown clearly. The values of first detected crystallisation are noted in the text. Orange, blue, grey and yellow curves correspond to increasing smoothing respectively.

There is a useful feature of turbidimeters which is very helpful for this determination, namely that they are commonly refractive index sensitive. This means that as the temperature is dropping the turbidity also drops marginally, so often leaving a minimum value, which can be taken as the crystallisation point. This is shown in Figure 4, although it should be noted that the actual curve is compound specific, as well as being dependent on the optical geometry of the turbidity probe. The lowest value of turbidity occurs at point 264 , which is also the last point beyond which there is no lower succeeding value, so is taken as the point of first detectable crystallisation.

The more scientific way to assess the beginning of crystallisation is to average the baseline over an area where one can be confident that no crystallisation is taking place, see Figure 6 , and to calculate the standard deviation (STDEV function in Microsoft Excel software). Then the first value above the deviation (or the last one at it) is taken as the start of crystallisation. The first value at or above the average value which does not have a succeeding lower value could also be used.

\section{INSERT FIGURE 6 HERE}

Figure 6. Statistical Determination of point of crystal detection. The average value and standard deviations from that value of the base line calculated from the first 400 points are shown by the maroon, green and purple lines. Point 493 where the turbidity curve in blue first exceeds the value of the average plus the 
standard deviation is taken as the crystallisation point.

These different methods will give marginally different crystallisation points, but provided they are used consistently and the cooling ramp is slow, they will all give results of equal applicability. In our experience the extra work that these statistically based approaches involves is not worthwhile. Similar values are obtained by the simpler and quicker visual assessment method. This opinion is based on the analysis of several thousand cooling crystallisations as well as by the data of Table 1 . Seeded crystallisation presents a further problem, although it has the advantage of diminishing the variation which is characteristic of unseeded nucleation ${ }^{4}$. When seeds are added there is an immediate variation of the baseline due to the presence of those seeds. Since they are often larger than the first detected crystals from homogenous nucleation, the baseline becomes correspondingly noisier as crystals pass in and out of the detector. The gently rising baseline due to crystal growth is generally shallower than that of unseeded crystallisation and its duration longer, because nucleation is occurring at a higher temperature with less thermodynamic driving force. If during the addition of seeds the turbidity probe is disturbed, another complexity in determining the change of background occurs. All these factors are likely to lead to more difficulty in the assessment of the crystallisation point. The proliferation point is unaffected by such considerations.

\section{Improving the clarity of solutions.}

One method of freeing solutions of potential heterogeneous nucleation particles is to perform a preliminary slight crystallisation followed by a filtration. The filtered solution should then be free of the unwanted active nuclei. This procedure is due to Mullin ${ }^{\S}$. It is useful in studies designed to separate homogenous and heterogeneous nucleation but is incompatible with usual crystallisation procedures. Ultrafiltration is generally more applicable when clean solutions are appropriate.

\section{Determining the temperature at which crystal dissolution is complete.}

As previously stated, it is generally considered that this should be easier to measure reliably than crystallisation temperatures. The methods described for the determination of the first crystallisation point can be used in reverse. Either the last point above the succeeding steady base line can be taken as the dissolution point; or for noisier traces, the last point for which there is no preceding lower value. Again, for noisy traces, averaging may help. The point at which the turbidity curve reaches the average base line or the average baseline plus its statistical deviation can be used exactly as described for the point of first crystal detection. Again, our experience is that it gives no more consistent results than those obtained by the simple analysis of the approach of the crystallisation curve to the base line. The effect of the refractive index on the turbidity seems to result more often in the point at which there is no preceding lower value also being the low point of the curve, as is seen the crystallisation process in Figure 4. There does not appear to be any reason why it should occur more often after the crystallisation than before it, since both ramp rates were always the same.

Another unexpected influence on the dissolution point may be the temperature of onset of crystallisation, shown in Figure 7.

\section{INSERT FIGURE 7 HERE}

Figure 7. The surprising influence of the crystallisation temperature on the subsequent crystallisation curve. Three consecutive crystallisation cycles are shown under identical temperature ramps. The later crystallisation results in less turbidity and even in marginally earlier dissolution. Temperature ramp in red, three successive turbidity cycles in orange, purple and blue.

The onset temperature may vary from run to run $^{2}$. This is shown by 3 successive unseeded cycles of D,L-malic acid crystallisation in figure 7 under identical temperature ramps. It may be found surprising that a delayed crystallisation can have such a continuing effect culminating with a small but measurable effect on the point of dissolution. The earliest crystallising run, curve in yellow, had a dissolution temperature of $39.98^{\circ} \mathrm{C}$, the intermediate run in purple a dissolution temperature of $39.84^{\circ} \mathrm{C}$ and the run in turquiose which seeded later, a dissolution temperature of $39.65^{\circ} \mathrm{C}$.

There are two probable reasons for any effect of the initial crystallisation temperature on the subsequent turbidity and dissolution. If the crystallisation is delayed then the turbidity is 
reduced leading to earlier detection of dissolution. See the later discussion of Figure 10 for elaboration of this. The later crystallisation also results in more rapid proliferation of particles under the greater thermodynamic driving force, leading to smaller particles. These dissolve without the kinetic delay occurring for large particles ${ }^{3}$.

If the crystal proliferation point is recorded, then the elbow of the dissolution should be used in order to maintain the correct MZW, although this refinement was not noted at the time the turbidity curves were analysed.

\section{Determination of the dissolution point in the presence of polymorphic change.}

If a minor transformation to a polymorph with a lower solubility occurs within the time scale of a crystallisation then it may not be realised that the dissolution of that polymorph rather than that of the supposed form is being measured. The detectability of the end-point may depend critically on the extent of the polymorphic transformation. For 6 years we struggled to obtain consistent results from the dissolution of $D, L-m a l i c$ acid before noticing that some minor polymorphic transition was often taking place. By manipulating the residence times at appropriate temperatures it was then possible to obtain clear evidence of the transformation (Figure 8) or even obtain the crystallisation curve of either polymorph free from the other.

\section{INSERT FIGURE 8 HERE}

Figure 8. Two consecutive cycles of the unseeded crystallisation of d,I-malic acid.

The metastable polymorph crystallises, as shown by the orange curve (even when seeded with crystals of the stable form). It subsequently begins to dissolve, but simultaneously is transforming into the stable form. Hence a pair of peaks is generated in each cycle, the first from the metastable crystallisation and partial dissolution and the second from the stable form. In the second cycle the lower temperature means that more of the initial product is formed, so has a higher turbidity. The different temperature ramps shown in blue and different crystallisation temperatures result in different amounts of polymorphic transformation, possibly due to the formation of larger crystals in the second cycle.
In such cases there is no ambiguity, but if a curve such as that shown in Figure $9 a$ or $9 \mathrm{~b}$ is obtained, then it may not be realised that what is measured is not the dissolution supposed. Turbidity curves are rarely symmetrical, and if a curve such as that of Figure 9a is observed, the shape may be ascribed to changes of aggregation, agglomeration ${ }^{15}$, crystal size or crystal shape of the solid phase at the higher temperature.

\section{INSERT FIGURE 9a HERE}

Figure 9a. D,I-malic turbidity curve showing some transformation. The shape of the turbidity curve may readily be erroneously ascribed to Ostwald ripening ${ }^{13,16}$ or to particle agglomeration ${ }^{15}$.

\section{INSERT FIGURE 9b HERE}

Figure 9b. D,I-malic acid turbidity curve showing minimum transformation. The arrow highlights the minor hump due to a small amount of transformation. The transformation is easily missed and the true position of the dissolution point is difficult to determine.

The tail of the curve, such as that of Figure $9 \mathrm{~b}$, may easily be overlooked. If it is seen, it may be thought to be due to some larger, slowly dissolving crystals rather than a pale imitation of that of another species. As Figure 10 shows, the ideal dissolution point becomes increasingly difficult to measure when the concentration of the species diminishes. If the ramp rate is reduced in order to deal with a tail, when the real cause is polymorphic transformation which must be to a more stable and so less soluble form, then more transformation will be encouraged and the tail will lengthen rather than shorten. Judging by our experience, the reality of the polymorphic change during crystallisation may not be recognised.

\section{INSERT FIGURE 10 HERE}

Figure 10. The effect of reducing intensity of turbidity curves on the crystallisation and dissolution points. The blue curve is full intensity, the orange is half, the grey a quarter, the yellow an eighth and the dark blue a sixteenth. 
The curves of Figure 10 were generated from a 30-point smoothing of the full intensity curve to give virtually noise free curves and then the full noise added back to each curve. The noise was generated by subtracting the smoothed curve from the original blue curve. So the final result is curves of decreasing intensity, but with identical noise levels. Although the crystallisation and dissolution points are changed significantly, the metastable zone width is barely altered, see Table 2 and discussion.

\begin{tabular}{|l|l|l|l|l|l|}
\hline Intensity $(\%)$ & 100 & 50 & 25 & 12.5 & 6.25 \\
\hline Crystallisation & 18.8 & 18.7 & 18.6 & 18.3 & 18.1 \\
Temperature $\left({ }^{\circ} \mathrm{C}\right)$ & 4 & 3 & 2 & 9 & 7 \\
\hline Dissolution & 41.8 & 41.7 & 41.5 & 41.3 & 39.0 \\
Temperature $\left({ }^{\circ} \mathrm{C}\right)$ & 7 & 0 & 7 & 4 & 6 \\
\hline MZW $\left({ }^{\circ} \mathrm{C}\right)$ & 23.0 & 22.9 & 22.9 & 22.9 & 20.8 \\
& 3 & 7 & 5 & 5 & 9 \\
\hline
\end{tabular}

Table 2. Crystallisation and dissolution temperatures and metastable zone widths $(\mathrm{MZW})$ in ${ }^{\circ} \mathrm{C}$ taken from Figure 10.

Figure 10 and Table 2 show that the metastable zone width is remarkably robust. Any method of detection, or any method of assessment of the crystallisation and dissolution points which takes those points to higher turbidities, will alter both the recorded crystallisation and recorded dissolution temperature. Provided the temperature ramps around the crystallisation and dissolution temperatures are the same, the metastable zone width will remain the same. Only if the recorded point has slipped on to a different ramp, as has the dissolution value for the one-sixteenth dilution in Table 2, will a different metastable zone width be noted.

\section{Relationship of the temperature of crystal proliferation and the time to crystal proliferation}

Just as there is a relationship between the temperature of crystal detection at constantly decreasing temperature and the time to crystal detection at constant temperature ${ }^{17}$, so the proliferation point can be measured either by decreasing temperature or by time to proliferation at constant temperature ${ }^{18}$. Under cooling conditions the determination of timeto-crystallisation at temperatures just above the normal crystallisation temperature is difficult because the delay time is so short. Typical programmable water baths either approach the set temperature semiexponentially, or with overshoot. Neither of these cooling regimes is compatible with accurate results. At higher temperatures, the times increase dramatically and the measurement of crystallisation point is generally less critical than in cooling crystallisations. One may then be confident that any variation is due to the inherent irreproducibility of crystallisation, rather than to errors in assessment.

\section{Conclusions}

For work in which crystallisation and dissolution temperatures are only being used in order to establish conditions for larger scale operation, or to draw an approximate solubility and crystallisation curve over a range of concentrations, the information provided here will provide assurance that whatever method of assessment is used, it will be adequate. What is surprising is the disconnect between the small variation between the various assessment methods described here of crystallisation and dissolution points and the variability in practice of repeated crystallisation cycles. This suggests that it is the inherent variability of the crystallisation process rather than its measurement which leads to the requirement of multiple determinations. It is hoped that the data and discussion in this paper will assist those studies, such as those of crystallisation mechanisms, in which the determinations are critical.

\section{Conflicts of interest}

There are no conflicts to declare.

\section{Acknowledgements}

Several undergraduate and masters students contributed practical work to this study, however we particularly acknowledge Esther Jones and Przemyslaw Ludwik Falak.

\section{Notes and references}

¥ Van Land and Wienck ${ }^{19}$ have claimed that sodium chloride nucleates first at a size of $50 \mu \mathrm{m}$. Recent studies ${ }^{20}$ have shown that the growth to visible sizes for sodium chloride is within a fraction of a second and so is hardly observable by eye. The accession and integration time for turbidimeters is typically 20 seconds, so only turbidity in the obscuration region could be 
observed for sodium chloride. Soluble salts typically grow rapidly to visible sizes after crystallisation.

¥ The crystallisation of magnesium sulfate probably follows Ostwald's Rule and so it is the hexahydrate which first nucleates ${ }^{20-22}$, even when seeded with the heptahydrate ${ }^{23-24}$. However, the change to heptahydrate is normally rapid so interference by hexahydrate is rarely encountered.

$\S$ This is true only if the particle size is sufficiently large, that is larger than about a micron. In the size region here light obscuration is dominant, therefore dependent on area rather than Rayleigh or Mie scattering ${ }^{8}$. If the point of crystal detection is checked by cross-illumination it will be found that it is at least as sensitive as turbidimetry and that the crystals are already quite large.

$\S \S$ We are indebted to Prof. Roger Davey of the University of Manchester for this unpublished information about the removal of seeding particles from solution.

1. R.S. Harner, R.J. Briggs, J.E. Hitt, P.A. Larsen and T.C. Frank, Org. Process Res. Dev., 2009, 13, 114124

2. A.R.Parsons, S.N. Black and R. Colling,

Trans/ChemE, 2003, 81, 700-701

3. J.W. Mullin, Crystallisation, $2^{\text {nd }}$ ed., Butterworths. London, 1972

4. S.S Kadam, S.A. Kulkani, R.C. Ribiera, A.J. Stankiewicz, J.H. ter Horst, and J.H. Kramer, Chem. Eng. Sci., 2012, 72, 911-919

5. T. Threlfall, Analyst, 1995, 120, 2435-2460

6. T. Threlfall, and S. Coles, CrystEngComm, 2016, 18, 369-378

7. D. Maes, Do protein crystals and aggregates go with the flow? Presentation at 50th British Association of Crystal Growth conference, London, 9-11 ${ }^{\text {th }}$ July, 2019.
8. A. Chianese and H.J.M. Kramer, Industrial Crystallization Process Monitoring and Control, Wiley-VCH, Weinheim, 2012; G. Oster and A.W. Pollister, Physical Techniques in Biological Research. Vol. 1, Optical Techniques. Academic Press, N.Y., 1955

9. J. Garside and M.A. Larson, J. Cryst. Growth, 1978, 43, 694-704

10. Z. Dai, F. Zhang, A. Kan, G. Ruan, F. Yan, N. Bhandari, Z. Zhang, Y. Liu. A. Y-T. Lu, G. Deng and M.B. Tomson, Ind. Eng. Chem. Res., 2019, 58, 10864-10874

11. H.H. Ting and W.L. McCabe, Ind. Eng. Chem., 1934, 26, 1201-1207

12. S.J. Coles, R. De'Ath and T. Threlfall, Org. Process Res. Dev., 2013, 17, 578-584

13. R.J. Davey and J. Garside, From Molecules to Crystallizers, Oxford Science Publications, 1999.

14. D.G. Bowers, K.M. Braithwaite, W.A.M. Nimmo-Smith, and G.W. Graham., Esturarine, Coastal and Shelf Sci., 2011, 91, 341-350

15. O.D. Linnikov, Russ. Chem. Rev. 2014, 83, 343404.

16. R.B. Fischer, Anal. Chem., 1951, 23, 1667-1671; I.M. Kolthoff, Analyst, 1952, 77, 1000-1011; M. Kahlweit, Adv. Coll. Interface Sci., 1975, 5, 1-35

17. S. Chen, H. Xi and L. Yu, J. Amer. Chem. Soc., 2005, 127, 17439-17444

18. H. Yang and A.J. Florence, CrystEngComm, 2017, 19, 3966-3978

19. C.M. van Land and B.G. Wienk, Industrial Crystallisation, ed. J.W. Mullin, Plenum Press, New York, 1976, pp. 51-60 
20. N. Shahidzadeh, University of Amsterdam, Personal communication.

21. T. Threlfall, Org. Process Res. Dev., 2003, 7, 1017-1027

22. W. Ostwald, Z. Phys. Chem., 1897, 22, 289-330

23. S.J. Coles and T. Threlfall, CrystEngComm, 2014, 16, 4355-4361

24. L. Yu, J. Amer. Chem. Soc., 2003, 125, 6380-

6381 